Case Report

\title{
Branch Retinal Artery Occlusion after Percutaneous Coronary Intervention
}

\author{
Tommaso La Macchia, ${ }^{1}$ Remo Albiero $\mathbb{D}^{2},{ }^{2}$ Tommaso Invernizzi, ${ }^{3}$ Giorgia Ceravolo, ${ }^{4}$ \\ and Ida Ceravolo 5 \\ ${ }^{1}$ Department of Clinical and Experimental Medicine, Unit of Cardiology, University of Messina, Via C. Valeria, 98125 Messina, Italy \\ ${ }^{2}$ Interventional Cardiology Unit-Sondrio Hospital, Via Stelvio, 25, 23100 Sondrio, Italy \\ ${ }^{3}$ Ophthalmology Unit-Sondrio Hospital, Via Stelvio, 25, 23100 Sondrio, Italy \\ ${ }^{4}$ Department of Human Pathology and Evolutive Age "Gaetano Barresi", University of Messina, Via C. Valeria, 98125 Messina, Italy \\ ${ }^{5}$ Department of Clinical and Experimental Medicine, University of Messina, Via C. Valeria, 98125 Messina, Italy
}

Correspondence should be addressed to Remo Albiero; albiero@panvascular.com

Received 23 March 2021; Accepted 16 April 2021; Published 27 April 2021

Academic Editor: Expedito E. Ribeiro

Copyright (C) 2021 Tommaso La Macchia et al. This is an open access article distributed under the Creative Commons Attribution License, which permits unrestricted use, distribution, and reproduction in any medium, provided the original work is properly cited.

\begin{abstract}
We report a case of branch retinal artery occlusion (BRAO) that occurred after percutaneous coronary intervention (PCI). A 59year-old man with no other previous diseases presented visual acuity deterioration in the left eye 24 hours after PCI. Fundus examination revealed ischemia at the temporal branch of the retinal artery associated with inner layer edema. Prompt treatment was performed with ocular digital massage and paracentesis of the anterior chamber. However, at discharge, the patient had a persistent visual loss with a central scotoma that persisted at 35-day follow-up without improvement of the visual acuity. The patient did not suffer from any other systemic complications. Retinal infarction should be considered a potential complication of PCI. Patients and health care providers should be aware of any visual signs. Permanent visual disability can be prevented by immediate diagnosis and prompt intervention.
\end{abstract}

\section{Introduction}

Percutaneous coronary intervention (PCI) is currently indicated for the management of patients presenting with coronary artery disease (CAD). Advances in technology, development of more potent and effective antiplatelet therapy, and judicious use of PCI are increasing the safety of the procedure. However, major complications during and after PCI still occur that can be related to the access site, intubation of the coronary artery ostia, or the intervention itself. The most common problems occur in the context of coronary intubation and target vessel or site intervention [1]. However, different organs including renal, brain, eye, and gastrointestinal systems could be targets of thromboembolic events during PCI or cardiac catheterization [2]. In particular, the association with some retinal alterations has been occasionally reported [3-9]. We report a case of branch retinal artery occlusion (BRAO) that occurred 24 hours after PCI.

\section{Case Report}

In November 2020, a 59-year-old man, an ex-smoker with negative previous medical history of heart disease, was admitted to the emergency department of Sondrio Hospital (Sondrio, Italy), 48 hours after the onset of episodes of rest angina associated with atypical angina symptoms (jaw pain in the dental arches).

Admission ECG showed a heart rate of $95 \mathrm{bpm}$ and a slight ST segment depression in the lateral leads. Measurement of cardiac biomarkers showed a rise in highsensitivity troponin I (hs-cTnI, $142 \mathrm{ng} / \mathrm{L}$ ). Double antiplatelet 


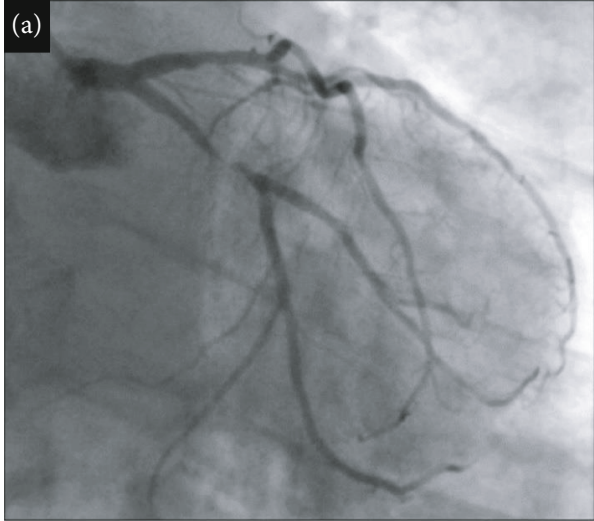

(a)

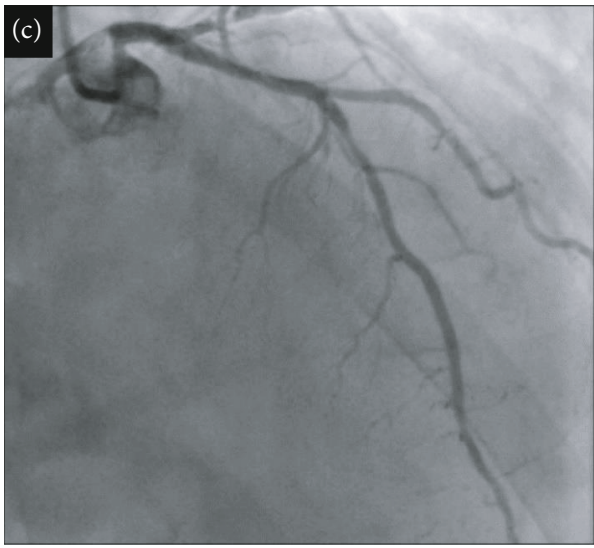

(c)

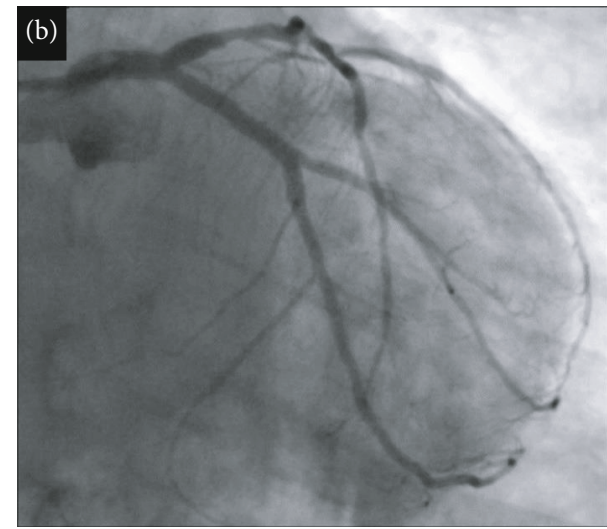

(b)

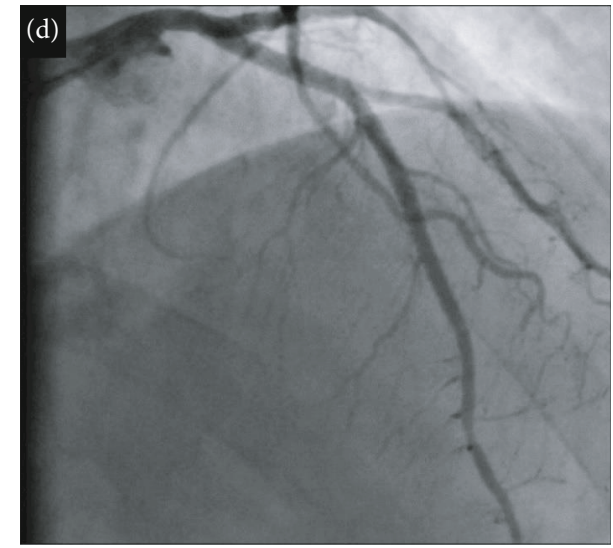

(d)

FIGURE 1: The angiogram shows 2-vessel CAD (coronary artery disease) with subocclusion of the proximal circumflex coronary artery (LCx) (a) and critical stenosis of the proximal and middle anterior descending coronary artery (LAD) (c). Good final result after PCI with implantation of a DES (drug-eluting stent) in the proximal-middle LCx at the bifurcation with the MO1 branch (b) and two contiguous DES on the proximal-middle LAD (d).

therapy (DAPT) with aspirin $100 \mathrm{mg}$ /day and clopidogrel $75 \mathrm{mg} /$ day was started on admission, and the same day, the patient underwent coronary angiography by right transradial access. The angiogram showed 2-vessel CAD (coronary artery disease) with subocclusion of the proximal circumflex coronary artery (LCx) and critical stenosis of the proximal and middle anterior descending coronary artery (LAD) (Figures 1(a) and 1(c)). After administration of heparin $100 \mathrm{U} / \mathrm{kg}$, ad hoc PCI was performed with implantation of a drug-eluting stent (DES) in the proximal-middle LCx at the bifurcation with the MO1 branch and two contiguous stents on the proximal-middle LAD, with a good final angiographic result on both vessels (Figures 1 (b) and 1(d)).

Twenty-four hours after PCI, the patient reported the onset of a left central scotoma and an ophthalmological evaluation was requested. The left visual acuity was reduced to $0.7 \log$ MAR with a central scotoma. There was no alteration in the anterior segment. Intraocular pressure (IOP) was $10 \mathrm{mmHg}$. Fundus examination revealed a white embolus in the temporal branch of the central retinal artery (Figure 2). A computerized visual field (Humphrey II 740 Visual Field, Carl Zeiss Meditec AG, Jena, Germany) revealed a paracentral visual defect (Figure 3(a)). Moreover,

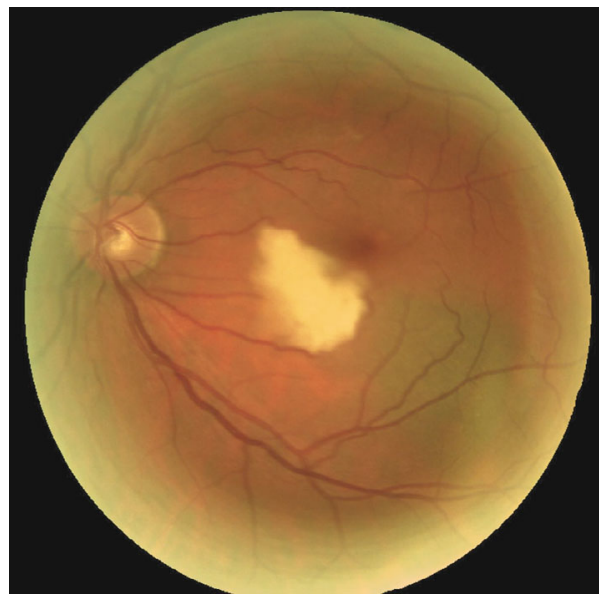

FIGURE 2: Fundus photograph of the left eye branch retinal artery occlusion (BRAO) showing white pallor at the inferior temporal branch of the central retinal artery.

spectral domain-optical coherence tomography (SD-OCT) (RS-3000 Nidek, Gamagori, Japan) was performed which showed inner layer edema in the temporal sector (Figure 3(b)). The right eye was normal. The diagnosis was 

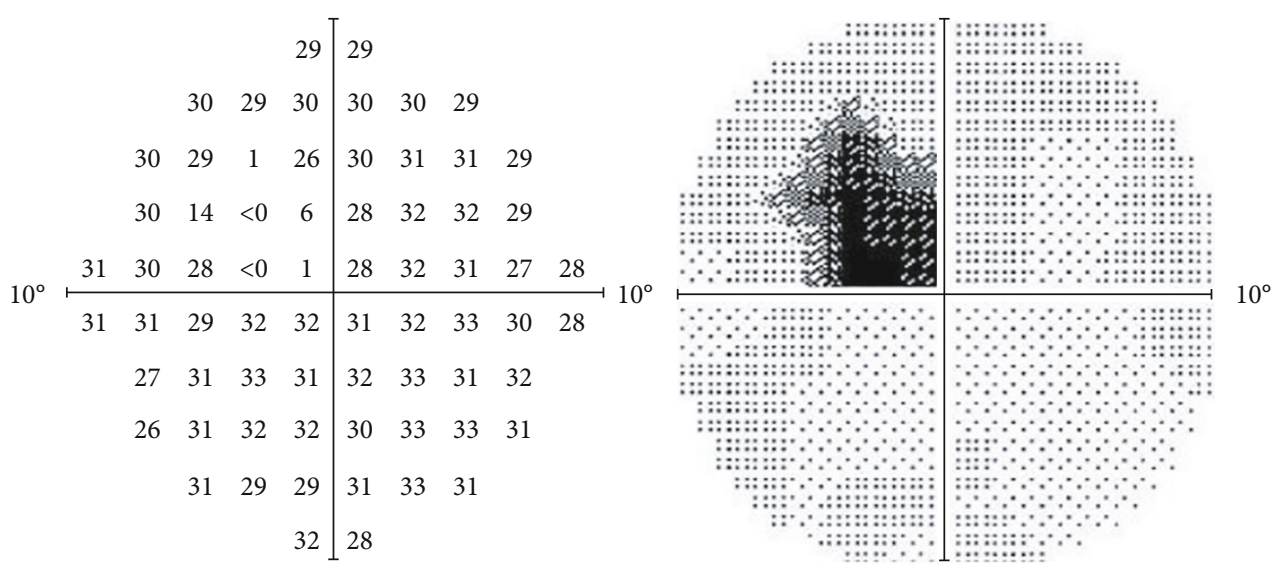

(a)

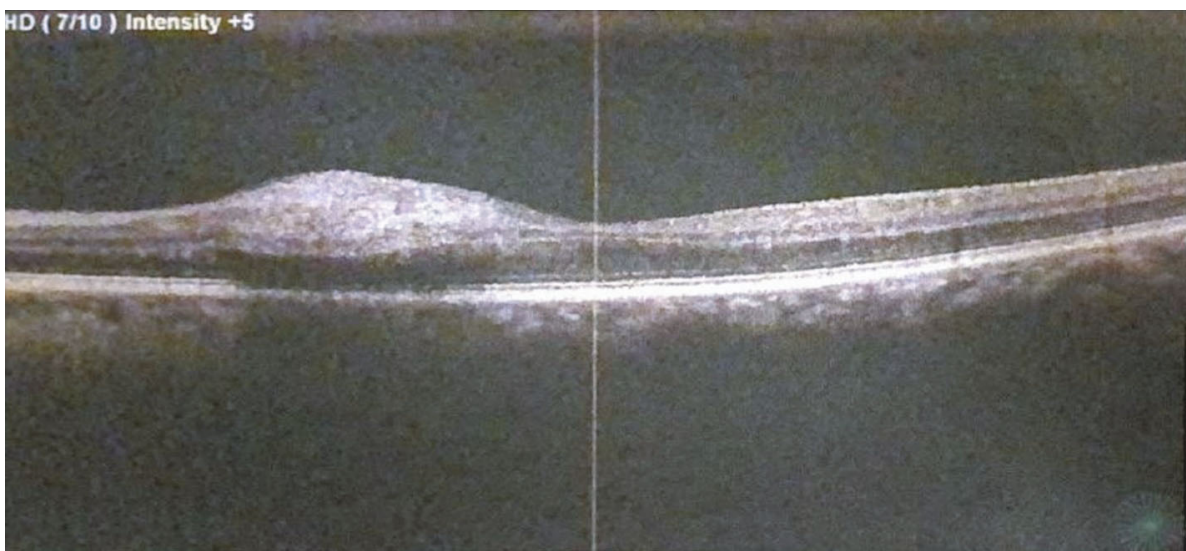

(b)

FIgure 3: (a) Visual field examination demonstrating defects in the $5^{\circ}$ temporal in the left eye. (b) Optical coherence tomography (OCT) images of the left eye showing temporal inner layer edema.

consistent with BRAO. Immediate conventional treatment of BRAO was performed, with ocular digital massage and paracentesis of the anterior chamber. Complete neurological examination was normal. The patient underwent supraaortic trunks echo-color Doppler (TSA) which showed bilaterally diffuse hyperplasia affecting the common carotid, the carotid bulb, and the internal and external carotid in the absence of hemodynamically significant flow accelerations. It showed no morphological alterations of the supra-aortic vessels. No other systemic complications occurred during hospitalization. At discharge, the patient had a persistent visual loss with a central scotoma that persisted at 35-day follow-up. In addition, follow-up OCT showed persistent macular edema. This study followed the tenets of the Declaration of Helsinki, and written consent was obtained from the patient.

\section{Discussion}

Branch retinal artery occlusion (BRAO) is one common cause of retinal vascular disease; it is caused by a reduction in arterial blood flow, resulting in retinal ischemia which can lead to severe vision loss and visual field defects despite therapy. Commonly, it is associated with systemic risk factors including hypertension, diabetes, carotid artery disease, and atherosclerosis. Few studies suggest that different retinal complications could be observed after coronary angiography procedures. Eye involvement includes central retinal artery occlusion (CRAO) [3, 4], branch retinal artery occlusion (BRAO) [5-7], or cilioretinal artery occlusion [8]. In addition, retinal embolisms following coronary catheterization can be clinically silent with any visual impairment. Moreover, it has been found that the risk factor for retinal emboli can be significantly associated with patient age, hypertension, and operator expertise [9]. Our patient had no additional risk factors. In the current case, the concomitance between the onset of eye disease and the coronary procedure suggests a correlation between the two conditions.

An immediate treatment is required if the occlusion occurs within 24 hours from the procedure. Conventional therapy consists in the reduction of intraocular pressure with hypotensive drugs, digital massage of the eyelid, and anterior chamber paracentesis. In some cases, thrombolytics can be infused into the carotid artery, or a laser embolectomy can be performed. The goal of the treatment is reperfusion of the blocked retinal vessels particularly when it is done within $6 \mathrm{~h}$, because retinal ischemic damage after $240 \mathrm{~min}$ is normally permanent [10]. However, treatments rarely improve 
visual acuity, and in BRAO, the visual field damage is typically permanent. Furthermore, in rare case, the condition could be complicated with retinal or iris neovascularization leading to glaucoma or vitreous hemorrhage.

\section{Conclusion}

With the growing occurrence of PCI, all physicians involved in patient care should be worried in keeping special attention towards ocular signs. Visual symptoms and retinal artery occlusions caused by embolization in patients after PCI could occur even 24 hours after catheterization. Prompt diagnosis and early treatment may help improve the outcome and preserve vision deterioration.

\section{Data Availability}

No data were used to support this study.

\section{Additional Points}

Learning Objective. Retinal infarction should be considered a potential complication of PCI with the risk of permanent visual disability that can be prevented by immediate diagnosis and prompt intervention. Patients and health care providers should be aware of any visual signs after PCI.

\section{Conflicts of Interest}

The authors declare that there is no conflict of interest.

\section{References}

[1] Y. R. Manda and K. M. Baradhi, Cardiac Catheterization Risks and Complications, StatPearls Publishing LLC, Treasure Island, FL, USA, 2021.

[2] M. Tavakol, S. Ashraf, and S. J. Brener, "Risks and complications of coronary angiography: a comprehensive review," Global Journal of Health Science, vol. 4, no. 1, pp. 65-93, 2012.

[3] P. Kraśnicki, E. Proniewska-Skretek, Z. Mariak, A. Dubicki, G. Mezyński, and S. Dobrzycki, "Embolic central retinal artery occlusion as a complication of percutaneous coronary angioplasty-case report," Klinika Oczna, vol. 110, no. 1-3, pp. 6466, 2008.

[4] J. Selton, T. Maalouf, A. Olivier, R. Anxionnat, M. Angioi, and K. Angioi, "Central retinal artery occlusion following coronary angiography: a case report," Journal Français d'Ophtalmologie, vol. 34, no. 3, pp. 181-185, 2011.

[5] G. D. Kymionis, M. K. Tsilimbaris, E. B. Christodoulakis, and I. G. Pallikaris, "Late onset branch retinal artery occlusion following coronary angiography," Acta Ophthalmologica Scandinavica, vol. 83, no. 1, pp. 122-123, 2005.

[6] K. C. Chan, D. J. Wu, K. C. Ueng et al., "Branch retinal artery occlusion after diagnostic cardiac catheterization," Japanese Heart Journal, vol. 43, no. 2, pp. 193-196, 2002.

[7] M. D. O'Neill, T. Akerele, and M. Dancy, "Branch retinal artery occlusion during coronary angiography," Postgraduate Medical Journal, vol. 81, no. 961, p. 710, 2005.

[8] A. Loewenstein, M. Goldstein, A. Roth, and M. Lazar, "Cilioretinal artery occlusion during coronary catheterization," Acta
Ophthalmologica Scandinavica, vol. 77, no. 6, pp. 717-718, 1999.

[9] J. Kojuri, M. Mehdizadeh, H. Rostami, and D. Shahidian, "Clinical significance of retinal emboli during diagnostic and therapeutic cardiac catheterization in patients with coronary artery disease," BMC Cardiovascular Disorders, vol. 11, no. 1, p. 5, 2011.

[10] Y. M. Hsien, M. Mustapha, J. C. Hamzah, O. Maskon, C. C. Ken, and C. H. Hamdi, "Why can't I see after my heart is fixed: a case series of ocular complications after cardiac intervention," BMC Ophthalmology, vol. 16, no. 1, p. 32, 2016. 